\title{
The geometric realization of Wall obstructions by nilpotent and simple spaces
}

\author{
By GUIDO MISLIN \\ ETH-Z, 8092 Zürich
}

(Received 12 March 1979)

Introduction. Let $\pi$ denote a finite group. It is well known that every element of the projective class group $K_{0} \mathbb{Z} \pi$ may be realized as Wall obstruction of a finitely dominated complex with fundamental group $\pi$ (ef. (13)). We will study two subgroups $N_{0} \mathbb{Z} \pi$ and $N \mathbb{Z} \pi$ of $K_{0} \mathbb{Z} \pi$, which are closely related to the Wall obstruction of nilpotent spaces. If the group $\pi$ is nilpotent and if $S$ denotes the set of elements $x \in K_{0} \mathbb{Z} \pi$ which occur as Wall obstructions of nilpotent spaces, then

$$
N_{0} \mathbb{Z} \pi \subset S \subset N \mathbb{Z} \pi \text {. }
$$

It turns out that in many instances one has $N_{0} \mathbb{Z} \pi=N \mathbb{Z} \pi$ (cf. Section 3 ) and one obtains hence new information on $S$. The main theorem $(2 \cdot 4)$ provides a systematic way of constructing finitely dominated nilpotent (or even simple) spaces with non-vanishing Wall obstructions.

1. The groups $T \mathbb{Z} \pi$ and $N \mathbb{Z} \pi$. If $\pi$ denotes a finite group then one defines $T \mathbb{Z} \pi \subset K_{0} \mathbb{Z} \pi$ to be the subgroup consisting of all elements of the form $[(k, N)]-[\mathbb{Z} \pi]$, where $N=\Sigma x$, $x \in \pi$, and $(k, N)$ is the projective ideal in $\mathbb{Z} \pi$ generated by $N$ and an integer $k$ prime to $\operatorname{card}(\pi)$. The group $T \mathbb{Z} \pi$ is known to be trivial if $\pi$ is cyclic (9). On the other hand $T \mathbb{Z} \pi \neq 0$ if $\pi$ contains a noncyclic subgroup of odd order (11). $T \mathbb{Z} \pi$ is completely known for $\pi$ a $p$-group (10).

It is convenient to think of $K_{0} \mathbb{Z} \pi$ to be generated by $\pi$-modules $M$ of type $F P$ and to write $[M]$ for the element $\Sigma(-1)^{i}\left[P_{i}\right] \in K_{0} \mathbb{Z} \pi$, if

$$
0 \rightarrow P_{n} \rightarrow P_{n-1} \rightarrow \ldots \rightarrow P_{0} \rightarrow M \rightarrow 0
$$

is a projective resolution of finite type. For instance, if $k$ is prime to card $(\pi)$ one has an exact sequence $0 \rightarrow \mathbb{Z} \pi \rightarrow(k, N) \rightarrow \mathbb{Z} / k \rightarrow 0$ and hence

$$
[(k, N)]-[\mathbb{Z} \pi]=[\mathbb{Z} / k] \in K_{0} \mathbb{Z} \pi,
$$

where $\mathbb{Z} / k$ is considered as a trivial $\pi$-module (cf. (4)). If $\pi \neq\{1\}$ then every trivial $\pi$-module of type $F P$ is necessarily finite and of order prime to $\operatorname{card}(\pi)$. We can then identify $T \mathbb{Z} \pi$ with the subgroup of $K_{0} \mathbb{Z} \pi$ consisting of all elements representable in the form [ $M]$, where $M$ is a trivial $\pi$-module of type $F P$.

In view of the applications we have in mind, we will define more general subgroups $N_{i} \mathbb{Z} \pi \subset K_{0} \mathbb{Z} \pi$ in a similar way.

Definition 1.1. Let $\pi$ be a finite non-trivial group. Then $N_{i} \mathbb{Z} \pi \subset K_{0} \mathbb{Z} \pi$ is the sub-group consisting of all elements of the form $\Sigma(-1)^{k}\left[P_{k}\right]$, where $P=\left\{P_{k}\right\}$ is a 
projective complex of finite type whose homology groups $H_{k}(P)$ are all nilpotent $\pi$-modules and for which $H_{j}(P)=0$ for $j>i$. Furthermore, $N \mathbb{Z} \pi=\cup N_{i} \mathbb{Z} \pi$ and for $\pi=\{1\}$ we define $N \mathbb{Z} \pi=N_{i} \mathbb{Z} \pi=0$ for all $i$.

To see that $N_{i} \mathbb{Z} \pi$ is indeed a subgroup for $\pi \neq\{1\}$, it suffices to check that all elements of $N_{i} \mathbb{Z} \pi$ are of finite order ( $N_{i} \mathbb{Z} \pi$ is obviously closed under addition). But this amounts to showing that $\Sigma(-1)^{k} \operatorname{rank}\left(P_{k}\right)=0$ for $P=\left\{P_{k}\right\}$ as in 1.1. But this follows immediately from the isomorphism

$$
H_{k}(P) \otimes \mathbb{Q} \stackrel{\cong}{\rightarrow} H_{k}(P) \otimes_{\pi} \mathbb{Q}
$$

which holds, since $H_{k}(P)$ is a nilpotent $\pi$-module, and from the equalities

$$
\Sigma(-1)^{k} \operatorname{rank}\left(P_{k}\right)=\Sigma(-1)^{k} \operatorname{dim}_{\mathbb{Q}}\left(H_{k}(P) \otimes_{\pi} \mathbb{Q}\right)=\frac{1}{|\pi|} \Sigma(-1)^{k} \operatorname{dim}_{\mathbb{Q}}\left(H_{k}(P) \otimes \mathbb{Q}\right) .
$$

By definition $N_{0} \mathbb{Z} \pi$ consists of elements $x=[M]$, where $M$ is a nilpotent $\pi$-module of type $F P$. If $P$ is as in $1 \cdot 1$ and $H_{j}(P)=0$ for $j>0$ then $H_{0}(P)=M$ is a finite module, since

$$
\Sigma(-1)^{k} \operatorname{rank}\left(P_{k}\right)=\operatorname{dim}_{\mathbb{Q}}\left(M \otimes_{\pi} \mathbb{Q}\right)=0 .
$$

Clearly, this $M$ is also cohomologically trivial, since it is of type $F P$.

Corollary 1.2. The subgroup $N_{0} \mathbb{Z} \pi \subset K_{0} \mathbb{Z} \pi$ consists of all elements $x=[M] \in K_{0} \mathbb{Z} \pi$, where $M$ is a finite, nilpotent, and cohomologically trivial $\pi$-module.

This is clear from the above, since a finite $M$ which is cohomologically trivial is of type $F P$ (and even of projective dimension $\leqslant 1$ by $(8)$ ).

In particular we see that $T \mathbb{Z} \pi \subset N_{0} \mathbb{Z} \pi \subset N \mathbb{Z} \pi$. The following example will illustrate that in general however $T \mathbb{Z} \pi \neq N_{0} \mathbb{Z} \pi$.

Lemma 1·3. If $\pi$ is cyclic of order 15 , then $N_{0} \mathbb{Z} \pi$ is of order two.

Proof. Choose a map $\pi \rightarrow$ Aut $(\mathbb{Z} / 9)$ which maps on to the subgroup of order 3 . This defines a nilpotent $\pi$-module $M$ with underlying abelian group $\mathbb{Z} / 9 . M$ is a nilpotent, cohomologically trivial $\pi$-module and $M$ generates $\widetilde{K}_{0} \mathbb{Z} \pi \cong \mathbb{Z} / 2$ (cf. (5), Lemma $2 \cdot 8$ ). Hence $N_{0} \mathbb{Z} \pi$ is of order two.

Remark. Let $D \mathbb{Z} \pi$ denote the kernel of the $\operatorname{map} K_{0} \mathbb{Z} \pi \rightarrow K_{0} \overline{\mathbb{Z} \pi}$, induced by including $\mathbb{Z} \pi$ into a maximal $\mathbb{Z}$-order $\overline{\mathbb{Z}} \pi$ in $\mathbb{Q} \pi$. If $\pi$ is nilpotent, then

$$
N \mathbb{Z} \pi \subset D \mathbb{Z} \pi \text {. }
$$

This is proved in (12) for $\pi$ cyclic and in (7) for a general nilpotent $\pi$. An example is given in (7) to show that in general $N \mathbb{Z} \pi \neq D \mathbb{Z} \pi$, even if $\pi$ is cyclic.

2. The realization theorem. All spaces we consider are supposed to be pointed connected $C W$-complexes; $X$ denotes the universal covering space of a space $X$. As usual a homology class is called spherical if it lies in the image of the Hurewicz homomorphism. First, we will describe a particular way of killing certain spherical classes.

Lemma 2-1. Let $X$ be an n-dimensional $C W$-complex and let $P \subset H_{n} \tilde{X}$ denote a projective $\pi_{1} X$-module consisting of spherical classes. Denote by $\phi: L \rightarrow H_{n} \tilde{X}$ a map from a 
free $\pi_{1} X$-module $L$ with basis $\left\{b_{\alpha}, \alpha \in I\right\}$, such that $\phi(L)=P$. Then one can form a new complex

$$
X^{\prime}=X \cup\left(\coprod_{\alpha \in I} e_{\alpha}^{n+1}\right)
$$

of dimension $n+1$ such that

(1) there is a commutative diagram

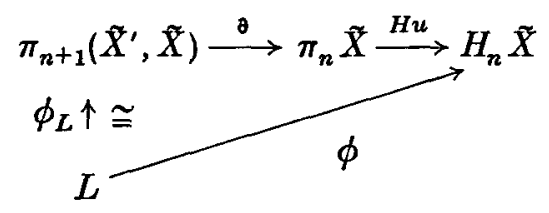

(2)

$$
H_{i} \tilde{X}^{\prime} \cong \begin{cases}H_{i} \tilde{X} & \text { if } i \neq n, n+1 \\ \left(H_{i} \tilde{X}\right) / P & \text { if } i=n \\ \operatorname{Ker} \phi & \text { if } i=n+1\end{cases}
$$

(3) $H_{n+1} \tilde{X}^{\prime}$ is projective and spherical (i.e. it consists entirely of spherical classes).

Proof. Since $P$ is projective, one can find $\bar{P} \subset \pi_{n} \tilde{X}$ such that $\bar{P}$ is mapped isomorphically onto $P$ by the Hurewicz homomorphism. Hence we can choose $\bar{\phi}: L \rightarrow \bar{P}$ to obtain a commutative diagram

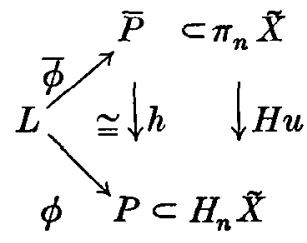

Denote by pr: $\hat{X} \rightarrow X$ the projection. We attach $(n+1)$-cells to $X$ using the maps $\operatorname{pr}\left(\bar{\phi} b_{\alpha}\right), \alpha \in I$, and obtain $X^{\prime}=X \cup\left(\amalg e_{\alpha}^{n+1}\right)$. It is immediate that $\phi$ lifts to an isomorphism $\phi_{L}$ giving rise to diagram (1). We consider now the diagram obtained by mapping the homotopy sequence of $\left(\tilde{X}^{\prime}, \tilde{X}\right)$ into the homology sequence of this pair:

$$
\begin{gathered}
\pi_{n+1} \tilde{X}^{\prime} \stackrel{a}{\rightarrow} \pi_{n+1}\left(\tilde{X}^{\prime}, \tilde{X}\right) \stackrel{\partial_{\pi}}{\rightarrow} \pi_{n} \tilde{X} \\
\downarrow \downarrow \downarrow \downarrow \\
\downarrow \\
H_{n+1} \tilde{X}^{\prime} \stackrel{\beta}{\rightarrow} H_{n+1}\left(\tilde{X}^{\prime}, \tilde{X}\right) \stackrel{\partial_{H}}{\rightarrow} H_{n} \tilde{X} \rightarrow H_{n} \tilde{X}^{\prime}
\end{gathered}
$$

Then $\operatorname{im} \partial_{\pi}=\bar{P}$ and hence $\operatorname{im} \partial_{H}=P$. Therefore $H_{n} \bar{X}^{\prime} \cong\left(H_{n} \tilde{X}\right) / P$. Note also that $\alpha\left(\pi_{n+1} \tilde{X}^{\prime}\right)$ is mapped isomorphically onto $\beta\left(H_{n+1} \tilde{X}^{\prime}\right) \cong H_{n+1} \tilde{X}^{\prime}$, since $\bar{P}$ is mapped isomorphically onto $P$. Hence $H_{n+1} \tilde{X}^{\prime} \cong \operatorname{Ker} \partial_{n} \cong \operatorname{Ker} \phi$ and $\pi_{n+1} \tilde{X}^{\prime} \rightarrow H_{n+1} \tilde{X}^{\prime}$ is onto. Therefore (2) and (3) hold.

Theorem 2.2. Let $X$ be a connected $C W$-complex of dimension $n>1$ and let $M$ be a $\pi_{1} X$-module of cohomological dimension $\leqslant 1$. Then there is a space $Y$ obtained from $X$ by attaching cells of dimension $\geqslant n$, such that

$$
H_{i} \tilde{Y}=\left\{\begin{array}{lll}
H_{i} \tilde{X} & \text { if } & i \neq n \\
\left(H_{n} \tilde{X}\right) \oplus M & \text { if } & i=n
\end{array}\right.
$$


Furthermore, if $X$ is finitely dominated and $M$ of type $F P$, then $Y$ can be chosen to be finitely dominated; the reduced Wall obstructions of $X$ and $Y$ are then related by

$$
\tilde{w} Y=\tilde{w} X+(-1)^{n}[M] \in \tilde{K}_{0} \mathbb{Z} \pi
$$

Proof. Choose a free resolution

$$
\ldots \rightarrow L_{n+i} \stackrel{\phi_{n+1}}{\longrightarrow} L_{n+i-1} \rightarrow \ldots \rightarrow L_{n+1} \stackrel{\phi_{n+1}}{\longrightarrow} L_{n} \rightarrow \rightarrow M .
$$

(If $M$ is a type $F P$, we choose a free resolution of finite type.) Since proj. $\operatorname{dim} M \leqslant 1$, im $\left(\phi_{n+i}\right)$ is projective for all $i \geqslant 1$. We construct $Y$ inductively as follows. Let $Y^{n}=X \vee B$, where $B$ is a bouquet of $n$-spheres corresponding to a basis of $L_{n}$. Then $H_{n} \tilde{Y}^{n} \cong\left(H_{n} \tilde{X}\right) \oplus L_{n}$ and $H_{n} \tilde{Y}^{n}$ contains a spherical projective submodule $P$ isomorphic to im $\left(\phi_{n+1}\right)$. Attaching $(n+1)$-cells to $Y^{n}$ with respect to the map $L_{n+1} \rightarrow H_{n} \tilde{Y}^{n}$ corresponding to $\phi_{n+1}$, we obtain by the previous Lemma a new space $Y^{n+1}$ with

$$
H_{i} \tilde{Y}^{n+1}= \begin{cases}H_{i} \tilde{X} & \text { if } i \neq n, n+1 \\ \left(H_{n} \tilde{X}\right) \oplus M & \text { if } i=n \\ \operatorname{Ker} \phi_{n+1} & \text { if } i=n+1 .\end{cases}
$$

Since $\operatorname{Ker} \phi_{n+1} \cong H_{n+1} \tilde{Y}^{n+1}$ is projective and spherical (Lemma 2.1) we can kill this group using $L_{n+2} \rightarrow H_{n+1} \tilde{Y}^{n+1}$. By repeating this construction we obtain spaces $Y^{n+k}$, $k \geqslant 1$, and we can form $Y=\cup Y^{n+k}$. By construction, $\tilde{Y}$ has the homology groups claimed in the theorem. Furthermore, the cellular chain complex of $\tilde{Y}$ is isomorphic to the complex

$$
\ldots \rightarrow L_{n+1} \rightarrow L_{n+i-1} \rightarrow \ldots \rightarrow L_{n} \oplus C_{n} \tilde{X} \rightarrow C_{n-1} \tilde{X} \rightarrow \ldots,
$$

where $C \tilde{X}$ is the cellular chain complex of $\tilde{X}$. Since the complex

$$
\ldots \rightarrow L_{n+i} \rightarrow L_{n+i-1} \rightarrow \ldots \rightarrow L_{n+1} \rightarrow \operatorname{im} \phi_{n+1}
$$

is contractible, it follows that $Y$ is a retract of $Y^{n+1}$. Hence $Y$ is finitely dominated, if $X$ is finitely dominated and $M$ of type $F P$. From the definition of the Wall obstruction it is immediate that

$$
\begin{aligned}
\tilde{w}(Y) & =\sum_{i=0}^{n}(-1)^{i}\left[\bar{C}_{i} X\right]+(-1)^{n+1}\left[\operatorname{im} \phi_{n+1}\right] \\
& =\tilde{w}(X)+(-1)^{n}[M]
\end{aligned}
$$

where $\bar{C} \tilde{X}$ is a chain complex of type $F P$, chain homotopy equivalent to $C \tilde{X}$.

Before we apply this Theorem to the construction of certain nilpotent spaces, we need the following elementary lemma.

Lemma 2-3. Let $\pi$ be a finite group. Then there exists a finite complex $X$ with $\pi_{1} X \cong \pi$ and Euler characteristic $\mathcal{X}(X)=0$, such that all covering transformations $t: \tilde{X} \rightarrow \bar{X}$ are homotopic to the identity.

Proof. Choose an embedding $\pi \subset S U(k)$. Then $X=S U(k) / \pi$ has the desired properties.

Note that $X=S U(k) / \pi$ is nilpotent, if $\pi$ is a nilpotent group, and it is a simple space, in case $\pi$ is abelian. 
We can now prove our main theorem.

THEOREM 2.4. Let $\pi$ be a finite nilpotent group and let $x \in N_{0} \mathbb{Z} \pi \subset K_{0} \mathbb{Z} \pi$. Then there exists a finitely dominated nilpotent space $Y$ with fundamental group $\pi$ and Wall obstruction $w(Y)=x$. If $x$ lies in $T \mathbb{Z} \pi$ and $\pi$ is abelian, then $Y$ may be chosen simple.

Proof. Let $x=[M]$ with $M$ a nilpotent $\pi$-module (trivial $\pi$-action in case $x \in T \mathbb{Z} \pi$ ). Choose $X=S U(k) / \pi$ as in the previous lemma (we may assume that $\operatorname{dim} X$ is even). Then, according to Theorem $2 \cdot 2$ we can construct a finitely dominated space $Y$ with $\pi_{1} Y \cong \pi_{1} X$ and

$$
H_{i} \tilde{Y}= \begin{cases}H_{i} \tilde{X} & \text { if } i \neq \operatorname{dim} X \\ \left(H_{i} \tilde{X}\right) \oplus M & \text { if } \quad i=\operatorname{dim} X\end{cases}
$$

It follows that $w Y=[M]=x$, since $X$ is finite with Euler characteristic 0. Moreover, $Y$ is nilpotent since its fundamental group is nilpotent and since $H_{i} \tilde{Y}$ is nilpotent for all $i$. In order to see that $Y$ is simple in case $\pi$ is abelian and $x \in T \mathbb{Z} \pi$, we prove the stronger result stating that, if $M$ is a trivial $\pi$-module, then all covering transformations $t: \tilde{Y} \rightarrow \tilde{Y}$ are homotopic to the identity. By the 'Hasse-Principle' for free maps (3) it suffices to show that the localizations $t_{p}: \tilde{Y}_{p} \rightarrow \tilde{Y}_{p}$ are homotopic to the identity for all primes $p$. If $p$ does not divide the order of $M$, then the inclusion $X \subset Y$ induces $H_{i} \tilde{X}_{p} \cong H_{i} \tilde{Y}_{p}$ and hence $X_{p} \simeq Y_{p}$ (the induced map of fundamental groups is certainly an isomorphism). Therefore $t_{p} \simeq \operatorname{Id} \tilde{Y}_{p}$ since the corresponding result is true for $X$ by construction. If $p$ divides the order of the trivial $\pi$-module $M$, then $p$ is necessarily prime to the order of $\pi$, since otherwise $M$ would not be cohomologically trivial. It follows therefore that the projection $\tilde{Y} \rightarrow Y$ induces a homotopy equivalence $\tilde{Y}_{p} \simeq Y_{p}$ if $p$ divides the order of $M$; clearly this implies that $t_{p} \simeq \operatorname{Id} \widetilde{Y}_{p}$ and hence the global map $t$ is homotopic to the identity (3).

Remark. If in Theorem $2 \cdot 4$ the assumption that $\pi$ be nilpotent is dropped, one can still construct the finitely dominated space $Y$ with $w(Y)=x \in N_{0} \mathbb{Z} \pi$. The space $Y$ will however in general only be homologically nilpotent in the sense that $\pi_{1} Y$ operates nilpotently on $H_{i} \tilde{Y}$ for all $i$.

Theorem $2 \cdot 4$ enables us to construct examples of the following types:

COROLLARY $2 \cdot 5$. (a) There exists a finitely dominated simple space with non-vanishing Wall obstruction.

(b) There exists a finitely dominated nilpotent space with cyclic fundamental group and non-vanishing Wall obstruction.

Proof. For $(a)$ choose any abelian group $\pi$ with $T \mathbb{Z} \pi \neq 0$ (e.g. $(\mathbb{Z} / p) \times(\mathbb{Z} / p) \times(\mathbb{Z} / p)$, $p$ any prime) and apply Theorem $2 \cdot 4$. Similarly, for $(b)$ we can choose any cyclic group $\pi$ with $N_{0} \mathbb{Z} \pi \neq 0$ (e.g. $\mathbb{Z} / 15$, cf. Lemma $1 \cdot 3$ ) and we obtain such an example by Theorem $2 \cdot 4$.

3. Some computations involving $N \mathbb{Z} \pi$. One can consider $N_{0} \mathbb{Z} \pi$ as a lower bound for the elements in $K_{0} \mathbb{Z} \pi$ which occur as Wall obstructions of finitely dominated homologically nilpotent space. Similarly, $N \mathbb{Z} \pi$ provides an upper bound for this set. The 
following examples show that for many groups one has $N_{0} \mathbb{Z} \pi=N \mathbb{Z} \pi$ and, indeed, we don't know of an example with $N_{0} \mathbb{Z} \pi \neq N \mathbb{Z} \pi$.

Our main tool will be a homomorphism

$$
T: U(\mathbb{Z}[1 / n]) \rightarrow K_{0} \mathbb{Z} \pi / T \mathbb{Z} \pi
$$

which was defined in (5) for groups $\pi$ with cyclic Sylow subgroups $(U(\mathbb{Z}[1 / n])$ the units in $\mathbb{Z}[1 / n]$ and $n=\operatorname{card}(\pi)$ ). For the following computation we will assume that $\pi$ is of square-free order $n$ (hence $T$ is defined). If $N=\Sigma x, x \in \pi$, the projection $\mathbb{Z} \pi \rightarrow \mathbb{Z} \pi / N$ induces an injective map

$$
\overline{p r}_{*}: K_{0} \mathbb{Z} \pi / T \mathbb{Z} \pi>\rightarrow K_{0}(\mathbb{Z} \pi / N) .
$$

It is convenient to describe $T$ by considering $K_{0}(\mathbb{Z} \pi / N)$ as the range of $T$. If $p$ is a prime dividing $n=\operatorname{card}(\pi)$ then the trivial $\pi$-module $\mathbb{Z} / p$ considered as a $\mathbb{Z} \pi / N$-module, is of type $F P$ with respect to the ring $\mathbb{Z} \pi / N$ and

$$
\overline{p r}_{*} T(p)=[\mathbb{Z} / p] \in K_{0}(\mathbb{Z} \pi / N) \text {. }
$$

Furthermore, $T(-1)=0$ (for details see (5)).

The connexion with $N \mathbb{Z} \pi$ is given by the next lemma.

Lemma 3.1. Let $\pi$ denote a group of square-free order $n$ and let $x \in N \mathbb{Z} \pi$. Let $P=\left\{P_{i}\right\}$ be a projective $\pi$-complex with $x=\Sigma(-1)^{i}\left[P_{i}\right]$ and $H_{i} P$ nilpotent for all $i$. Then

$$
\rho(P)=\operatorname{card} H_{\mathrm{ev}}\left(\mathbb{Z} \pi / N \otimes_{\pi} P\right) / \operatorname{card} H_{\text {odd }}\left(\mathbb{Z} \pi / N \otimes_{\pi} P\right)
$$

is a unit in $\mathbb{Z}[1 / n]$ and if $\bar{x}$ denotes the image of $x$ in $N \mathbb{Z} \pi / T \mathbb{Z} \pi$ then

$$
\bar{x}=T \rho(P) \in K_{0} \mathbb{Z} \pi / T \mathbb{Z} \pi .
$$

In particular one has $N \mathbb{Z} \pi / T \mathbb{Z} \pi \subset \operatorname{im}(T)$.

Proof. This result was proved in ((5), Section 3) in case $x=w X$, the Wall obstruction of a homologically nilpotent space $X$. The same proof works for an arbitrary $x \in N \mathbb{Z} \pi$.

Conollary 3.2. Let $\pi$ be of order $p$ or $2 p, p$ an arbitrary prime. Then $N Z \pi=0$.

Proof. If card $(\pi)=p$ with $p$ an arbitrary prime or if $\operatorname{card}(\pi)=2 p, p$ an odd prime, then $\operatorname{im}(T)=0$ by ((5), Theorem 2.5). Hence $N \mathbb{Z} \pi=T \mathbb{Z} \pi$ in these cases. But in both cases one has $T \mathbb{Z} \pi=0$ (cf. (11)). It remains to consider the case $\operatorname{card}(\pi)=4$. But it is well known that $\mathbb{K}_{0} \mathbb{Z} \pi=0$ if $\operatorname{card}(\pi)=4$. Hence the result follows.

THEOREM 3.3. If $\pi$ is a cyclic group of square-free order, then

$$
N_{0} \mathbb{Z} \pi=N \mathbb{Z} \pi=\operatorname{im}(T) .
$$

Proof. Note that $T \mathbb{Z} \pi=0$ for $\pi$ cyclic. Hence $N \mathbb{Z} \pi \subset \operatorname{im}(T)$ by Lemma $3 \cdot 1$ and it suffices therefore to show that $\operatorname{im}(T) \subset N_{0} \mathbb{Z} \pi$. If $p$ is a prime dividing card $(\pi)$ then $\overline{p r}_{*} T(p)=[\mathbb{Z} / p] \in K_{0}(\mathbb{Z} \pi / N)$. It remains to prove that there exist $x \in N_{0} \mathbb{Z} \pi$ with $\overline{p r}_{*} x=[\mathbb{Z} / p]$. If $x=[M], M$ a nilpotent $\pi$-module of projective dimension $\leqslant 1$, and if $0 \rightarrow P_{1} \rightarrow P_{0} \rightarrow M \rightarrow 0$ is a resolution of type $F P$, then by definition

$$
p r_{*} x=\left[\mathbb{Z} \pi / N \otimes_{\pi} P_{0}\right]-\left[\mathbb{Z} \pi / N \otimes_{n} P_{1}\right]
$$


But since $M$ is cohomologically trivial, one has

$$
\operatorname{Tor}_{1}^{\pi}(\mathbb{Z} \pi / N, M) \cong \operatorname{Ker}(M / I M \stackrel{N}{\rightarrow} M)=\hat{H}^{-1}(\pi, M)=0
$$

and therefore the sequence

$$
0 \rightarrow \mathbb{Z} \pi / N \otimes_{\pi} P_{1} \rightarrow \mathbb{Z} \pi / N \otimes_{\pi} P_{0} \rightarrow \mathbb{Z} \pi / N \otimes_{\pi} M \rightarrow 0
$$

is exact. Hence we can write

$$
\overline{p r}_{*}[M]=\left[\mathbb{Z} \pi / N \otimes_{\pi} M\right] .
$$

We will now construct for all prime divisors of $\operatorname{card}(\pi)$ nilpotent $\pi$-modules of type $F P$ with $\mathbb{Z} \pi / N \otimes_{n} M \cong \mathbb{Z} / p$. First consider the case of an odd prime $p$. Let $\mathbb{Z} / p$ act in a non-trivial way on $\mathbb{Z} / p^{2}$ and define a $\pi$-action on $\mathbb{Z} / p^{2}$ using a surjection $\pi \rightarrow \mathbb{Z} / p$. One verifies easily that the resulting $\pi$-module $M$ is nilpotent and cohomologically trivial. Furthermore, $\overline{p r}_{*}[M]=\left[\mathbb{Z} \pi / N \otimes_{\pi} M\right]=[M / N M]=[\mathbb{Z} / p]$. If $p=2$, one can use $\mathbb{Z} / 8$ as underlying abelian group for $M$, which one equips with a $\mathbb{Z} / 2$-action by mapping $a$ into $5 a, a \in \mathbb{Z} / 8$, and defining a $\pi$-module structure by means of a surjection $\pi \rightarrow \rightarrow \mathbb{Z} / 2$. Again one verifies that $\overline{p r}_{*}[M]=[\mathbb{Z} / 2]$. Hence im $(T) \subset N_{0} \mathbb{Z} \pi$ and the result follows.

For the groups of Theorem 3.3 we can obtain an upper bound for the order and the exponent of $N \mathbb{Z} \pi$ in terms of the Euler $\phi$-function $\phi(n)=$ card $(U(\mathbb{Z} / n))$ and the function $e(n)=($ exponent of $U(\mathbb{Z} / n))$.

Theorem 3.4. If $\pi$ is a cyclic group of square-free order $n$, then the order of

$$
N_{0} \mathbb{Z} \pi=N \mathbb{Z} \pi
$$

divides $\phi(n) / e(n)$ and its exponent divides e $(n)$.

Proof. Let $p$ be a prime which divides $n$ and let $\bar{\pi} \subset \pi$ be a subgroup of index $p$. The $[\mathbb{Z} / p] \in T \mathbb{Z} \bar{\pi} \subset K_{0} \mathbb{Z} \bar{\pi}$ is mapped to $\left[\mathbb{Z} \pi \otimes_{\pi} \mathbb{Z} / p\right]=[\mathbb{Z} / p[\pi / \bar{n}]] \in K_{0} \mathbb{Z} \pi$ by the map induced by $\bar{\pi} \subset \pi$ (one uses that $\operatorname{Tor}_{n}^{\frac{1}{2}}(\mathbb{Z} \pi, \mathbb{Z} / p)=0$ ). But if $M=\mathbb{Z} / p[\pi / \bar{\pi}]$ then $\mathbb{Z} \pi / N \otimes_{\pi} M=M / N M$ is a nilpotent $\pi$-module of cardinality $p^{p-1}$ and hence $\overline{p r}_{*}[M]=(p-1) \overline{p r}_{*} T(p) \in K_{0}(\mathbb{Z} \pi / N)$. Since $T \mathbb{Z} \bar{\pi}=0(\bar{\pi}$ is cyclic $),[M]=0$ and hence $(p-1) T(p)=0$. We obtain therefore a factorization

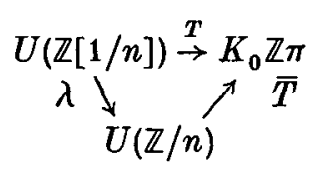

where $\lambda: U(\mathbb{Z}[1 / n]) \rightarrow U(\mathbb{Z} / n) \cong \Pi(\mathbb{Z} / p-1)$ is defined by $\lambda(-1)=0$ and $\lambda(p)=(0, \ldots, 0, \overline{1}, 0, \ldots 0)$ if $p$ divides $n$. The diagonal element $\Delta=(\overline{1}, \ldots, \overline{1})$ in $U(\mathbb{Z} / n) \cong \Pi(\mathbb{Z} / p-1)$ is mapped to 0 by $\bar{T}$, since $T(n)=0$ (cf. Theorem 2.5 of (5)). Moreover, $N_{0} \mathbb{Z} \pi=N \mathbb{Z} \pi=\operatorname{im}(T)$ by Theorem 3.3. Hence the exponent of $N \mathbb{Z} \pi$ divides the exponent of $U(\mathbb{Z} / n)$ and the order of $N \mathbb{Z} \pi$ divides $\phi(n) / e(n)$ which is the order of $U(\mathbb{Z} / n) /\langle\Delta\rangle$.

For example, if $\pi$ is a cyclic group of order $3 p, p$ a prime $>3$, then $\operatorname{card}(N \mathbb{Z} \pi) \leqslant 2$ since $\phi(3 p)=2(p-1)$ and $e(2 p)=p-1$. 
As a final example, we want to compute $N \mathbb{Z} \pi$ in case $\pi=M(p, q)$, the metacyclic group of square-free order $p q, p$ and $q$ odd primes and $q \mid p-1$, defined by

$$
M(p, q)=\left\langle x, y \mid x^{p}=y^{q}=1, y^{-1} x y=x^{r}\right\rangle,
$$

$r$ a primitive $q$ th root of $1 \bmod p$.

Theorem 3.5. Let $\pi=M(p, q)$. Then

$$
T \mathbb{Z} \pi=N_{0} \mathbb{Z} \pi=N \mathbb{Z} \pi \cong \mathbb{Z} / q .
$$

Proof. It has been shown in (6) that if $x \in K_{0} \mathbb{Z} \pi$ is the Wall obstruction of a homologically nilpotent space $X$ with fundamental group $M(p, q)$, then $x \in T \mathbb{Z} \pi$. The same argument shows that for an arbitrary $x \in N \mathbb{Z} \pi$ one has $x \in T \mathbb{Z} \pi$ and hence $N \mathbb{Z} \pi=T \mathbb{Z} \pi$. Furthermore, $T \mathbb{Z} \pi=\mathbb{Z} / q$ by (11).

One can combine the results of this section to obtain the following table for $N \mathbb{Z} \pi$, in case $\pi$ is a group of small, square-free order.

COROLLARY 3·6. Let $\pi$ be a group of square-free order $n<30$. Then

$$
N_{0} \mathbb{Z} \pi=N \mathbb{Z} \pi= \begin{cases}0 & \text { if } n \neq 15,21 \\ \mathbb{Z} / 2 & \text { if } n=15 \\ \mathbb{Z} / 3 & \text { if } n=21, \pi \text { noncyclic } \\ \mathbb{Z} / 2 \text { or } 0 & \text { if } n=21, \pi \text { cyclic. }\end{cases}
$$

\section{REFERENCES}

(1) Fröhlich, A. On the class group of integral group rings of finite abelian groups. I. Mathematika 16 (1969), 143-152.

(2) Fröhlich, A., Keating, M. E. and Wilson, S. M. J. The class groups of quaternion and dihedral 2-groups. Mathematika 21 (1974), 64-71.

(3) Hilton, P., Mistin, G., Roitberg, J. and Steiner, R. On free maps and free homotopies into nilpotent spaces. Springer Lecture Notes in Math. Vol. 673, 1977.

(4) Misiln, G. Finitely dominated nilpotent spaces. Ann. of Math. 103 (1976), 547-556.

(5) Mislin, G. Groups with cyclic Sylow subgroups and finiteness conditions for certain complexes. Comment. Math. Helv. 52 (1977), 373-391.

(6) Miscin, G. Finitely dominated complexes with metacyclic fundamental groups. Topology and Algebra, Proceedings of a Colloquium in honour of B. Eckmann, Monographie No. 26, L'Enseignement Mathématique 1978, 233-235.

(7) Miscin, G. and Varadarajan, K. The finiteness obstruction for nilpotent spaces lie in $\mathrm{D}(\mathbb{Z} \pi)$. Inventiones math. 53 (1979), 185-191.

(8) RrM, D. S. Modules over finite groups. Ann. of Math. 63 (1959), 700-712.

(9) SwaN, R. G. Periodic resolutions for finite groups. Ann. of Math. 72 (1960), 267-291.

(10) TAYLOR, M. J. Locally free class groups of prime power order. J. Algebra 50 (1978), 463-487.

(11) ULLOM, S. V. Nontrivial lower bounds for class groups of integral group rings. Illinois $J$. of Math. 20 (1976), 361-371.

(12) Varadarajan, K. Finiteness obstructions for nilpotent spaces. J. Pure and Appl. Algebra 12 (1978), 137-146.

(13) WALl, C. T. C. Finiteness conditions for CW-complexes. Ann. of Math. 81 (1965), 56-69. 